\title{
A study of a coupled system of Hadamard fractional differential equations with nonlocal coupled initial-multipoint conditions
}

\author{
Bashir Ahmad $^{1 *} \mathbb{D}$, Sotiris K. Ntouyas ${ }^{2,1}$, Ahmed Alsaedi ${ }^{1}$ and Amjad F. Albideewi
}

\author{
"Correspondence: \\ bashirahmad_qau@yahoo.com; \\ bahmad@kau.edu.sa \\ 'Nonlinear Analysis and Applied \\ Mathematics (NAAM)-Research \\ Group, Department of Mathematics, \\ Faculty of Science, King Abdulaziz \\ University, P.O. Box 80203, Jeddah \\ 21589, Saudi Arabia \\ Full list of author information is \\ available at the end of the article
}

\author{
Abstract \\ In this paper, we obtain the existence results for a coupled system of Hadamard \\ fractional differential equations supplemented with nonlocal coupled \\ initial-multipoint conditions via fixed point theorems. An example is constructed for \\ the illustration of the uniqueness result. \\ MSC: 34A08; 34A12; 34B15 \\ Keywords: Hadamard fractional integral; Hadamard fractional derivative; Nonlocal \\ conditions; Existence; Fixed point theorems
}

\section{Introduction}

Nonlocal boundary value problems have been extensively studied by many researchers. One can witness the theoretical development and applications of this class of problems in the related literature. The idea of nonlocal conditions dates back to the work of Hilb [1]. However, Bitsadze and Samarskii [2] presented the systematic investigation of spatial nonlocal problems. For motivation of nonlocal conditions, see $[3,4]$.

Fractional calculus, regarded as a generalization of classical calculus, deals with the differential and integral operators of noninteger order. One can find a detailed account of fractional-order differential equations in the monographs [5-9] and the references therein. On the other hand, several interesting results on Hadamard-type fractional differential equations, inclusions, and inequalities can be found in [10]. In [11], a monotone iterative method was applied to study the existence of positive solutions for Hadamard fractional differential equations complemented with nonlocal multi-point discrete and Hadamard integral boundary conditions. For application of Hadamard fractional differential equations, we refer the reader to the papers $[12,13]$.

Boundary value problems involving systems of fractional differential equations have also been studied by many researchers in view of their applications in the real world problems. It prompted many investigators to explore the theoretical aspects of fractional differential systems. For some recent results on Hadamard fractional differential systems, for instance, see [14-19].

(c) The Author(s) 2021. This article is licensed under a Creative Commons Attribution 4.0 International License, which permits use, sharing, adaptation, distribution and reproduction in any medium or format, as long as you give appropriate credit to the original author(s) and the source, provide a link to the Creative Commons licence, and indicate if changes were made. The images or other third party material in this article are included in the article's Creative Commons licence, unless indicated otherwise in a credit line to the material. If material is not included in the article's Creative Commons licence and your intended use is not permitted by statutory regulation or exceeds the permitted use, you will need to obtain permission directly from the copyright holder. To view a copy of this licence, visit http://creativecommons.org/licenses/by/4.0/. 
Recently, in [20], the authors derived existence results for a Hadamard-type fractional differential equation equipped with nonlocal initial condition given by

$$
\left\{\begin{array}{l}
{ }^{H} D^{q} x(t)=f(t, x(t)), \quad 1<t<T, 0<q \leq 1, \\
x(1)+\sum_{j=1}^{m} \zeta_{j} x\left(t_{j}\right)=0,
\end{array}\right.
$$

where ${ }^{H} D^{q}$ denotes the Hadamard fractional derivative of order $q, f:[1, T] \times \mathbb{R} \rightarrow \mathbb{R}, t_{j}$, $j=1,2, \ldots, m$ are given points with $1 \leq t_{1} \leq \cdots \leq t_{m}<T$ and $\zeta_{j}$ are real numbers such that

$$
1+\sum_{j=1}^{m} \zeta_{j} \neq 0
$$

The inclusions case of the problem (1) was also discussed in [20]. Problem (1) was studied for $q=1$ in [21], for time scales setting in [22], and for Caputo fractional differential equations in [23].

In the present paper, motivated by [20], we introduce and investigate the existence of solutions for a coupled system of nonlinear Hadamard type fractional differential equations

$$
\begin{cases}{ }^{H} D^{p} x(t)=f(t, x(t), y(t)), & 1<t<T, \\ { }^{H} D^{q} y(t)=g(t, x(t), y(t)), & 1<t<T, 0<p, q \leq 1,\end{cases}
$$

subject to nonlocal coupled initial-multipoint conditions:

$$
\left\{\begin{array}{l}
x(1)+\sum_{j=1}^{m} \alpha_{j} y\left(t_{j}\right)=0, \\
y(1)+\sum_{j=1}^{m} \beta_{j} x\left(t_{j}\right)=0,
\end{array}\right.
$$

where ${ }^{H} D^{p},{ }^{H} D^{q}$ denote the Hadamard fractional derivatives of orders $p$ and $q$, respectively, $f, g:[1, T] \times \mathbb{R}^{2} \rightarrow \mathbb{R}$ are Carathéodory functions, $t_{j}$ are given points with $1 \leq t_{1} \leq$ $\cdots \leq t_{m}<T$, and $\alpha_{j}, \beta_{j}$ are real numbers such that $1-\sum_{j=1}^{m} \alpha_{j} \sum_{j=1}^{m} \beta_{j} \neq 0$.

Existence results for the system (2)-(3) are proved by applying fixed point theorems. In Sect. 3, we discuss an existence result using the idea employed in [21-23], where the growth condition is split into two subintervals: one containing the points involved in the nonlocal condition, while the second deals with the rest of the interval. In Sect. 4, we present two more results for the system (2)-(3): an existence and uniqueness result is obtained by using Banach's fixed point theorem, while Leray-Schauder alternative is employed to obtain an existence result by assuming a growth condition on $f$ and $g$ on the whole interval.

\section{Preliminaries}

In this section, we introduce notation and definitions which are used throughout this paper. Let $X=\{x(t): x(t) \in C([1, T], \mathbb{R})\}$ be endowed with the norm $\|x\|=\|x\|_{[1, T]}=$ $\max _{t \in[1, T]}|x(t)|$. Obviously $(X,\|\cdot\|)$ is a Banach space. Also $Y=\{y(t): y(t) \in C([1, T], \mathbb{R})\}$ endowed with the norm $\|y\|=\|y\|_{[1, T]}=\max _{t \in[1, T]}|y(t)|$ is a Banach space. Then the product space $(X \times Y,\|(x, y)\|)$ is also a Banach space equipped with the norm $\|(x, y)\|=$ $\|x\|+\|y\|$. 
We denote by $L^{1}([1, T], \mathbb{R})$ the Banach space of measurable functions $x:[1, T] \rightarrow \mathbb{R}$, which are Lebesgue integrable, and normed by

$$
\|x\|_{L^{1}}=\int_{1}^{T}|x(t)| d t \quad \text { for all } x \in L^{1}([1, T], \mathbb{R}) .
$$

Let us recall some basic definitions on fractional calculus.

Definition 1 The Hadamard derivative of fractional order q for a function $g:[1, \infty) \rightarrow \mathbb{R}$ is defined as

$$
{ }^{H} D^{q} g(t)=\frac{1}{\Gamma(n-q)}\left(t \frac{d}{d t}\right)^{n} \int_{1}^{t}\left(\log \frac{t}{s}\right)^{n-q-1} \frac{g(s)}{s} d s, \quad n-1<q<n, n=[q]+1,
$$

where $[q]$ denotes the integer part of the real number $q$ and $\log (\cdot)=\log _{e}(\cdot)$.

Definition 2 The Hadamard fractional integral of order $q$ for a function $g$ is defined as

$$
I^{q} g(t)=\frac{1}{\Gamma(q)} \int_{1}^{t}\left(\log \frac{t}{s}\right)^{q-1} \frac{g(s)}{s} d s, \quad q>0,
$$

provided the integral exists.

In order to define the solution of problem (2)-(3), we consider the following lemma, concerning a linear variant of problem (2)-(3).

Lemma 1 Let $h, k \in C([1, T], \mathbb{R})$ and $Q:=1-\sum_{j=1}^{m} \alpha_{j} \sum_{j=1}^{m} \beta_{j} \neq 0$. Then the solution for the linear system of fractional differential equations

$$
\begin{cases}{ }^{H} D^{p} x(t)=h_{1}(t), & 1<t<T, \\ { }^{H} D^{q} y(t)=h_{2}(t), & 1<t<T, 0<p, q \leq 1,\end{cases}
$$

supplemented with nonlocal coupled initial-multipoint conditions in (3) is equivalent to the integral equations

$$
\begin{aligned}
x(t)= & \frac{1}{\Gamma(p)} \int_{1}^{t}\left(\log \frac{t}{s}\right)^{p-1} \frac{h_{1}(s)}{s} d s-\frac{1}{Q}\left[\sum_{j=1}^{m} \alpha_{j} \frac{1}{\Gamma(q)} \int_{1}^{t_{j}}\left(\log \frac{t_{j}}{s}\right)^{q-1} \frac{h_{2}(s)}{s} d s\right. \\
& \left.-\sum_{j=1}^{m} \alpha_{j} \sum_{j=1}^{m} \beta_{j} \frac{1}{\Gamma(p)} \int_{1}^{t_{j}}\left(\log \frac{t_{j}}{s}\right)^{p-1} \frac{h_{1}(s)}{s} d s\right]
\end{aligned}
$$

and

$$
\begin{aligned}
y(t)= & \frac{1}{\Gamma(q)} \int_{1}^{t}\left(\log \frac{t}{s}\right)^{q-1} \frac{h_{2}(s)}{s} d s-\frac{1}{Q}\left[\sum_{j=1}^{m} \beta_{j} \frac{1}{\Gamma(p)} \int_{1}^{t_{j}}\left(\log \frac{t_{j}}{s}\right)^{p-1} \frac{h_{1}(s)}{s} d s\right. \\
& \left.-\sum_{j=1}^{m} \alpha_{j} \sum_{j=1}^{m} \beta_{j} \frac{1}{\Gamma(q)} \int_{1}^{t_{j}}\left(\log \frac{t_{j}}{s}\right)^{q-1} \frac{h_{2}(s)}{s} d s\right] .
\end{aligned}
$$


Proof For some constant $x_{0}, y_{0} \in \mathbb{R}$, we have

$$
\begin{aligned}
& x(t)=\frac{1}{\Gamma(p)} \int_{1}^{t}\left(\log \frac{t}{s}\right)^{p-1} \frac{h_{1}(s)}{s} d s-x_{0}, \\
& y(t)=\frac{1}{\Gamma(q)} \int_{1}^{t}\left(\log \frac{t}{s}\right)^{q-1} \frac{h_{2}(s)}{s} d s-y_{0} .
\end{aligned}
$$

Then, we obtain

$$
\begin{aligned}
& x\left(t_{j}\right)=\frac{1}{\Gamma(p)} \int_{1}^{t_{j}}\left(\log \frac{t_{j}}{s}\right)^{p-1} \frac{h_{1}(s)}{s} d s-x_{0}, \\
& y\left(t_{j}\right)=\frac{1}{\Gamma(q)} \int_{1}^{t_{j}}\left(\log \frac{t_{j}}{s}\right)^{q-1} \frac{h_{2}(s)}{s} d s-y_{0} .
\end{aligned}
$$

Using (7) and (8) in the initial conditions (3), we obtain a system of equations in the unknown constants $x_{0}$ and $y_{0}$ given by

$$
\left\{\begin{array}{l}
x_{0}+\sum_{j=1}^{m} \alpha_{j} y_{0}=A_{1}, \\
\sum_{j=1}^{m} \beta_{j} x_{0}+y_{0}=A_{2},
\end{array}\right.
$$

where

$$
\begin{aligned}
& A_{1}=\sum_{j=1}^{m} \alpha_{j} \frac{1}{\Gamma(q)} \int_{1}^{t_{j}}\left(\log \frac{t_{j}}{s}\right)^{q-1} \frac{h_{2}(s)}{s} d s \\
& A_{2}=\sum_{j=1}^{m} \beta_{j} \frac{1}{\Gamma(p)} \int_{1}^{t_{j}}\left(\log \frac{t_{j}}{s}\right)^{p-1} \frac{h_{1}(s)}{s} d s .
\end{aligned}
$$

Solving the system (9) for $x_{0}$ and $y_{0}$, we find that

$$
x_{0}=\frac{1}{Q}\left[A_{1}-\sum_{j=1}^{m} \alpha_{j} A_{2}\right], \quad y_{0}=\frac{1}{Q}\left[A_{2}-\sum_{j=1}^{m} \beta_{j} A_{1}\right] .
$$

Substituting the values of $x_{0}$ and $y_{0}$ in (7), together with the notations (10), leads to the solution (5) and (6). The converse can be proved by direct computation. The proof is completed.

For convenience, we set

$$
\begin{aligned}
& B=1+|\eta| \sum_{j=1}^{m}\left|\alpha_{j}\right| \sum_{j=1}^{m}\left|\beta_{j}\right|, \quad C=|\eta| \sum_{j=1}^{m}\left|\alpha_{j}\right|, \quad D=|\eta| \sum_{j=1}^{m}\left|\beta_{j}\right|, \quad \eta=\frac{1}{Q}, \\
& m_{1}=\frac{B(\log T)^{p}}{\Gamma(p+1)}, \quad m_{2}=\frac{C(\log T)^{q}}{\Gamma(q+1)}, \quad n_{1}=\frac{B(\log T)^{q}}{\Gamma(q+1)}, \quad n_{2}=\frac{D(\log T)^{p}}{\Gamma(p+1)}, \\
& l_{1}=\frac{B}{\Gamma(p)} \int_{1}^{T}\left(\log \frac{T}{s}\right)^{p-1} \frac{k_{1}(s)}{s} d s, \quad l_{2}=\frac{C}{\Gamma(q)} \int_{1}^{T}\left(\log \frac{T}{s}\right)^{q-1} \frac{k_{2}(s)}{s} d s \\
& l_{3}=\frac{B}{\Gamma(q)} \int_{1}^{T}\left(\log \frac{T}{s}\right)^{q-1} \frac{k_{2}(s)}{s} d s, \quad l_{4}=\frac{D}{\Gamma(p)} \int_{1}^{T}\left(\log \frac{T}{s}\right)^{p-1} \frac{k_{1}(s)}{s} d s .
\end{aligned}
$$




\section{Existence result with mixed growth condition}

We assume that $f, g:[1, T] \times \mathbb{R}^{2} \rightarrow \mathbb{R}$ are Carathéodory functions and prove an existence result with mixed growth condition.

Definition 3 We say that $f, g:[1, T] \times \mathbb{R}^{2} \rightarrow \mathbb{R}$ are $L^{1}$-Carathéodory if

(i) $t \longmapsto f(t, x, y), t \longmapsto g(t, x, y)$ are measurable for each $(x, y) \in \mathbb{R}^{2}$;

(ii) $(x, y) \longmapsto f(t, x, y),(x, y) \longmapsto g(t, x, y)$ are continuous for almost all $t \in[1, T]$;

(iii) For each $\mu>0$, there exist $\sigma_{\mu}, \varrho_{\mu} \in L^{1}\left([1, T], \mathbb{R}^{+}\right)$such that $|f(t, x, y)| \leq \sigma_{\mu}$ for almost all $t \in[1, T]$ and for all $x, y \in \mathbb{R}^{+}$such that $\|x\| \leq \mu,\|y\| \leq \mu$, and $|g(t, x, y)| \leq \varrho_{\mu}$ for almost all $t \in[1, T]$ and for all $x, y \in \mathbb{R}^{+}$such that $\|x\| \leq \mu$, $\|y\| \leq \mu$.

In view of Lemma 1, we define an operator $\mathcal{F}: X \times Y \rightarrow X \times Y$ by

$$
\mathcal{F}(x, y)(t):=\left(\mathcal{F}_{1}(x, y)(t), \mathcal{F}_{2}(x, y)(t)\right)
$$

where

$$
\begin{aligned}
\mathcal{F}_{1}(x, y)(t)= & \frac{1}{\Gamma(p)} \int_{1}^{t}\left(\log \frac{t}{s}\right)^{p-1} \frac{f(s, x(s), y(s))}{s} d s \\
& -\eta\left[\sum_{j=1}^{m} \alpha_{j} \frac{1}{\Gamma(q)} \int_{1}^{t_{j}}\left(\log \frac{t_{j}}{s}\right)^{q-1} \frac{g(s, x(s), y(s))}{s} d s\right. \\
& \left.-\sum_{j=1}^{m} \alpha_{j} \sum_{j=1}^{m} \beta_{j} \frac{1}{\Gamma(p)} \int_{1}^{t_{j}}\left(\log \frac{t_{j}}{s}\right)^{p-1} \frac{f(s, x(s), y(s))}{s} d s\right], \quad t \in[1, T],
\end{aligned}
$$

and

$$
\begin{aligned}
\mathcal{F}_{2}(x, y)(t)= & \frac{1}{\Gamma(q)} \int_{1}^{t}\left(\log \frac{t}{s}\right)^{q-1} \frac{g(s, x(s), y(s))}{s} d s \\
& -\eta\left[\sum_{j=1}^{m} \beta_{j} \frac{1}{\Gamma(p)} \int_{1}^{t_{j}}\left(\log \frac{t_{j}}{s}\right)^{p-1} \frac{f(s, x(s), y(s))}{s} d s\right. \\
& \left.-\sum_{j=1}^{m} \alpha_{j} \sum_{j=1}^{m} \beta_{j} \frac{1}{\Gamma(q)} \int_{1}^{t_{j}}\left(\log \frac{t_{j}}{s}\right)^{q-1} \frac{g(s, x(s), y(s))}{s} d s\right], \quad t \in[1, T] .
\end{aligned}
$$

Note that the operators $\mathcal{F}_{1}$ and $\mathcal{F}_{2}$ given by (13) and (14) appear as sums of two integral operators: one of Fredholm type, whose values depend only on the restrictions of functions to $\left[1, t_{m}\right]$ and which is given by

$$
\mathcal{F}_{F_{1}}(x, y)(t)=\left\{\begin{array}{rlr}
\frac{1}{\Gamma(p)} \int_{1}^{t}\left(\log \frac{t}{s}\right)^{p-1} \frac{f(s, x(s), y(s))}{s} d s & \\
& -\eta\left[\sum_{j=1}^{m} \alpha_{j} \frac{1}{\Gamma(q)} \int_{1}^{t_{j}}\left(\log \frac{t_{j}}{s}\right)^{q-1} \frac{g(s, x(s), y(s))}{s} d s\right. & \\
& \left.-\sum_{j=1}^{m} \alpha_{j} \sum_{j=1}^{m} \beta_{j} \frac{1}{\Gamma(p)} \int_{1}^{t_{j}}\left(\log \frac{t_{j}}{s}\right)^{p-1 \frac{f(s, x(s), y(s))}{s}} d s\right], & \text { if } t<t_{m}, \\
\frac{1}{\Gamma(p)} \int_{1}^{t_{m}}\left(\log \frac{t_{m}}{s}\right)^{p-1} \frac{f(s, x(s), y(s))}{s} d s & \\
& -\eta\left[\sum_{j=1}^{m} \alpha_{j} \frac{1}{\Gamma(q)} \int_{1}^{t_{j}}\left(\log \frac{t_{j}}{s}\right)^{q-1} \frac{g(s, x(s), y(s))}{s} d s\right. & \\
& \left.-\sum_{j=1}^{m} \alpha_{j} \sum_{j=1}^{m} \beta_{j} \frac{1}{\Gamma(p)} \int_{1}^{t_{j}}\left(\log \frac{t_{j}}{s}\right)^{p-1 \frac{f(s, x(s), y(s))}{s}} d s\right], & \text { if } t \geq t_{m},
\end{array}\right.
$$


and the other of Volterra type, defined by

$$
\mathcal{F}_{V_{1}}(x, y)(t)= \begin{cases}0 & \text { if } t<t_{m}, \\ \frac{1}{\Gamma(p)} \int_{t_{m}}^{t}\left(\log \frac{t}{s}\right)^{p-1} \frac{f(s, x(s), y(s))}{s} d s, & \text { if } t \geq t_{m},\end{cases}
$$

depending on the restriction of function to $\left[t_{m}, T\right]$.

Similarly, $\mathcal{F}_{2}=\mathcal{F}_{F_{2}}+\mathcal{F}_{V_{2}}$, where

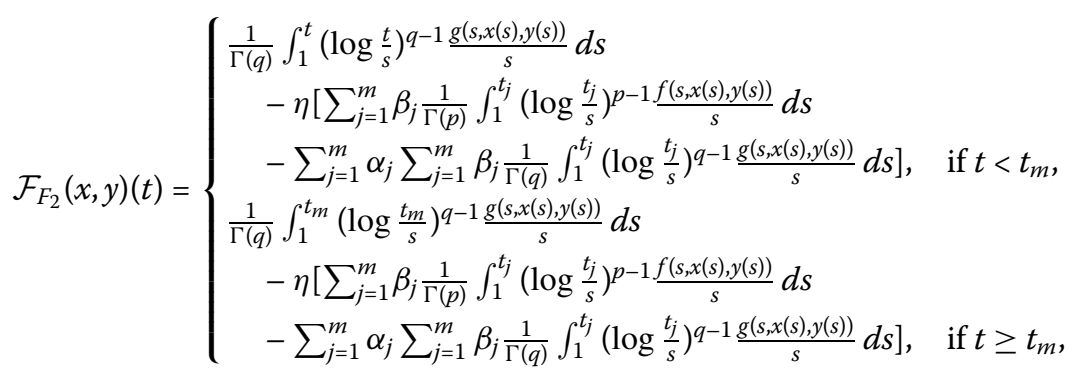

and the Volterra type operator is defined by

$$
\mathcal{F}_{V_{2}}(x, y)(t)= \begin{cases}0 & \text { if } t<t_{m}, \\ \frac{1}{\Gamma(q)} \int_{t_{m}}^{t}\left(\log \frac{t}{s}\right)^{q-1} \frac{g(s, x(s), y(s))}{s} d s, & \text { if } t \geq t_{m} .\end{cases}
$$

This allows us to split the growth condition on the nonlinear terms $f(t, x, y)$ and $g(t, x, y)$ into two parts, namely, for $t \in\left[1, t_{m}\right]$ and $t \in\left[t_{m}, T\right]$.

\section{Theorem 1 Assume that}

$\left(H_{1}\right) f, g:[1, T] \times \mathbb{R}^{2} \rightarrow \mathbb{R}$ are $L^{1}$-Carathéodory functions;

$\left(H_{2}\right)$ There exist continuous functions $\omega_{1}, \omega_{2}$ nondecreasing in their second argument, $k \in L^{1}\left[t_{m}, T\right]$, and nondecreasing functions $\Psi_{1}, \Psi_{2}: \mathbb{R}^{+} \rightarrow \mathbb{R}^{+}$such that

$$
\begin{aligned}
& |f(t, x, y)|= \begin{cases}\omega_{1}(t,|x|,|y|), & \text { if } t \in\left[1, t_{m}\right], \\
k(t) \Psi_{1}(|x|,|y|), & \text { if } t \in\left[t_{m}, T\right],\end{cases} \\
& |g(t, x, y)|= \begin{cases}\omega_{2}(t,|x|,|y|), & \text { if } t \in\left[1, t_{m}\right], \\
k(t) \Psi_{2}(|x|,|y|), & \text { if } t \in\left[t_{m}, T\right] ;\end{cases}
\end{aligned}
$$

$\left(H_{3}\right)$ There exists $R_{0}>0$ such that

$$
\begin{aligned}
\rho>R_{0} \Longrightarrow & \frac{B+D}{\Gamma(p)} \int_{1}^{t_{m}}\left(\log \frac{t_{m}}{s}\right)^{p-1} \frac{\omega_{1}\left(s,\left|\rho_{1}\right|,\left|\rho_{2}\right|\right)}{s} d s \\
& +\frac{B+C}{\Gamma(q)} \int_{1}^{t_{m}}\left(\log \frac{t_{m}}{s}\right)^{q-1} \frac{\omega_{2}\left(s,\left|\rho_{1}\right|,\left|\rho_{2}\right|\right)}{s} d s<\rho ;
\end{aligned}
$$


$\left(H_{4}\right) \lim \sup _{R \rightarrow \infty} \frac{R}{\Lambda_{1}+\Lambda_{2}}>1$, where

$$
\begin{aligned}
\Lambda_{1}= & \frac{B+D}{\Gamma(p)} \int_{1}^{t_{m}}\left(\log \frac{t}{s}\right)^{p-1} \frac{\omega_{1}\left(s, R_{0}, R_{0}\right)}{s} d s \\
& +\Psi_{1}(R, R) \frac{1}{\Gamma(p)} \int_{t_{m}}^{T}\left(\log \frac{T}{s}\right)^{p-1} \frac{k(s)}{s} d s \\
\Lambda_{2}= & \frac{B+C}{\Gamma(q)} \int_{1}^{t_{m}}\left(\log \frac{t}{s}\right)^{q-1} \frac{\omega_{2}\left(s, R_{0}, R_{0}\right)}{s} d s \\
& +\Psi_{2}(R, R) \frac{1}{\Gamma(q)} \int_{t_{m}}^{T}\left(\log \frac{t}{s}\right)^{q-1} \frac{k(s)}{s} d s .
\end{aligned}
$$

Then, problem (2)-(3) has at least one solution on $[1, T]$.

Proof We show that the solutions of (2)-(3) are a priori bounded. Let $(x, y)$ be a solution. Then, for $t \in\left[1, t_{m}\right]$, we have

$$
\begin{aligned}
& |x(t)|=\left|\lambda \mathcal{F}_{1}(x, y)(t)\right| \\
& =\lambda \mid \frac{1}{\Gamma(p)} \int_{1}^{t}\left(\log \frac{t}{s}\right)^{p-1} \frac{f(s, x(s), y(s))}{s} d s \\
& -\eta\left[\sum_{j=1}^{m} \alpha_{j} \frac{1}{\Gamma(q)} \int_{1}^{t_{j}}\left(\log \frac{t_{j}}{s}\right)^{q-1} \frac{g(s, x(s), y(s))}{s} d s\right. \\
& \left.-\sum_{j=1}^{m} \alpha_{j} \sum_{j=1}^{m} \beta_{j} \frac{1}{\Gamma(p)} \int_{1}^{t_{j}}\left(\log \frac{t_{j}}{s}\right)^{p-1} \frac{f(s, x(s), y(s))}{s} d s\right] \mid \\
& \leq \frac{1}{\Gamma(p)} \int_{1}^{t_{m}}\left(\log \frac{t_{m}}{s}\right)^{p-1} \frac{|f(s, x(s), y(s))|}{s} d s \\
& +|\eta|\left[\sum_{j=1}^{m}\left|\alpha_{j}\right| \frac{1}{\Gamma(q)} \int_{1}^{t_{m}}\left(\log \frac{t_{j}}{s}\right)^{q-1} \frac{|g(s, x(s), y(s))|}{s} d s\right. \\
& \left.+\sum_{j=1}^{m}\left|\alpha_{j}\right| \sum_{j=1}^{m}\left|\beta_{j}\right| \frac{1}{\Gamma(p)} \int_{1}^{t_{m}}\left(\log \frac{t_{j}}{s}\right)^{p-1} \frac{|f(s, x(s), y(s))|}{s} d s\right] \\
& \leq B \frac{1}{\Gamma(p)} \int_{1}^{t_{m}}\left(\log \frac{t_{m}}{s}\right)^{p-1} \frac{\omega_{1}(s,|x(s)|,|y(s)|)}{s} d s \\
& +C \frac{1}{\Gamma(q)} \int_{1}^{t_{m}}\left(\log \frac{t_{m}}{s}\right)^{q-1} \frac{\omega_{2}(s,|x(s)|,|y(s)|)}{s} d s,
\end{aligned}
$$

which, on taking the supremum for $t \in\left[1, t_{m}\right]$, yields

$$
\begin{aligned}
\|x\|_{\left[1, t_{m}\right]} \leq & \frac{B}{\Gamma(p)} \int_{1}^{t_{m}}\left(\log \frac{t_{m}}{s}\right)^{p-1} \frac{\omega_{1}\left(s,\|x\|_{\left[1, t_{m}\right]},\|y\|_{\left[1, t_{m}\right]}\right)}{s} d s \\
& +\frac{C}{\Gamma(q)} \int_{1}^{t_{m}}\left(\log \frac{t_{m}}{s}\right)^{q-1} \frac{\omega_{2}\left(s,\|x\|_{\left[1, t_{m}\right]},\|y\|_{\left[1, t_{m}\right]}\right)}{s} d s .
\end{aligned}
$$


Similarly,

$$
\begin{aligned}
\|y\|_{\left[1, t_{m}\right]} \leq & \frac{B}{\Gamma(q)} \int_{1}^{t_{m}}\left(\log \frac{t_{m}}{s}\right)^{q-1} \frac{\omega_{2}\left(s,\|x\|_{\left[1, t_{m}\right]},\|y\|_{\left[1, t_{m}\right]}\right)}{s} d s \\
& +\frac{D}{\Gamma(p)} \int_{1}^{t_{m}}\left(\log \frac{t_{m}}{s}\right)^{p-1} \frac{\omega_{1}\left(s,\|x\|_{\left[1, t_{m}\right]},\|y\|_{\left[1, t_{m}\right]}\right)}{s} d s .
\end{aligned}
$$

Let $\rho_{1}=\|x\|_{\left[1, t_{m}\right]}, \rho_{2}=\|y\|_{\left[1, t_{m}\right]}$. Then from (19) and (20), we deduce

$$
\left\{\begin{array}{l}
\rho_{1} \leq \frac{B}{\Gamma(p)} \int_{1}^{t_{m}}\left(\log \frac{t_{m}}{s}\right)^{p-1} \frac{\omega_{1}\left(s, \rho_{1}, \rho_{2}\right)}{s} d s+\frac{C}{\Gamma(q)} \int_{1}^{t_{m}}\left(\log \frac{t_{m}}{s}\right)^{q-1} \frac{\omega_{2}\left(s, \rho_{1}, \rho_{2}\right)}{s} d s \\
\rho_{2} \leq \frac{B}{\Gamma(q)} \int_{1}^{t_{m}}\left(\log \frac{t_{m}}{s}\right)^{q-1} \frac{\omega_{2}\left(s, \rho_{1}, \rho_{2}\right)}{s} d s+\frac{D}{\Gamma(p)} \int_{1}^{t_{m}}\left(\log \frac{t_{m}}{s}\right)^{p-1} \frac{\omega_{1}\left(s, \rho_{1}, \rho_{2}\right)}{s} d s .
\end{array}\right.
$$

Then

$$
\begin{aligned}
\rho= & \rho_{1}+\rho_{2} \\
\leq & \frac{B+D}{\Gamma(p)} \int_{1}^{t_{m}}\left(\log \frac{t_{m}}{s}\right)^{p-1} \frac{\omega_{1}\left(s,\left|\rho_{1}\right|,\left|\rho_{2}\right|\right)}{s} d s \\
& +\frac{B+C}{\Gamma(q)} \int_{1}^{t_{m}}\left(\log \frac{t_{m}}{s}\right)^{q-1} \frac{\omega_{2}\left(s,\left|\rho_{1}\right|,\left|\rho_{2}\right|\right)}{s} d s .
\end{aligned}
$$

Then, assumption $\left(H_{3}\right)$ guarantees that

$$
\rho \leq R_{0}
$$

Next, we let $t \in\left[t_{m}, T\right]$. Then

$$
\begin{aligned}
|x(t)|= & \left|\lambda \mathcal{F}_{1}(x, y)(t)\right| \\
= & \lambda \mid \frac{1}{\Gamma(p)} \int_{1}^{t}\left(\log \frac{t}{s}\right)^{p-1} \frac{f(s, x(s), y(s))}{s} d s \\
& -\eta\left[\sum_{j=1}^{m} \alpha_{j} \frac{1}{\Gamma(q)} \int_{1}^{t_{j}}\left(\log \frac{t_{j}}{s}\right)^{q-1} \frac{g(s, x(s), y(s))}{s} d s\right. \\
& \left.-\sum_{j=1}^{m} \alpha_{j} \sum_{j=1}^{m} \beta_{j} \frac{1}{\Gamma(p)} \int_{1}^{t_{j}}\left(\log \frac{t_{j}}{s}\right)^{p-1} \frac{f(s, x(s), y(s))}{s} d s\right] \mid \\
\leq & \frac{1}{\Gamma(p)} \int_{1}^{t_{m}}\left(\log \frac{t}{s}\right)^{p-1} \frac{\omega_{1}\left(s, R_{0}, R_{0}\right)}{s} d t \\
& +|\eta|\left[\sum_{j=1}^{m}\left|\alpha_{j}\right| \frac{1}{\Gamma(q)} \int_{1}^{t_{j}}\left(\log \frac{t_{j}}{s}\right)^{q-1} \frac{\omega_{2}\left(s, R_{0}, R_{0}\right)}{s} d t\right. \\
& \left.+\sum_{j=1}^{m}\left|\alpha_{j}\right| \sum_{j=1}^{m}\left|\beta_{j}\right| \frac{1}{\Gamma(p)} \int_{1}^{t_{j}}\left(\log \frac{t_{j}}{s}\right)^{p-1} \frac{\omega_{1}\left(s, R_{0}, R_{0}\right)}{s} d t\right] \\
& +\frac{1}{\Gamma(p)} \int_{t_{m}}^{t}\left(\log \frac{t}{s}\right)^{p-1} \frac{k(s) \Psi_{1}(|x(s)|,|y(s)|)}{s} d s
\end{aligned}
$$




$$
\begin{aligned}
\leq & \frac{B}{\Gamma(p)} \int_{1}^{t_{m}}\left(\log \frac{t}{s}\right)^{p-1} \frac{\omega_{1}\left(s, R_{0}, R_{0}\right)}{s} d s+\frac{C}{\Gamma(q)} \int_{1}^{t_{m}}\left(\log \frac{t}{s}\right)^{q-1} \frac{\omega_{2}\left(s, R_{0}, R_{0}\right)}{s} d s \\
& +\frac{1}{\Gamma(p)} \int_{t_{m}}^{t}\left(\log \frac{t}{s}\right)^{p-1} \frac{k(s) \Psi_{1}(|x(s)|,|y(s)|)}{s} d s \\
\leq & \frac{B}{\Gamma(p)} \int_{1}^{t_{m}}\left(\log \frac{t}{s}\right)^{p-1} \frac{\omega_{1}\left(s, R_{0}, R_{0}\right)}{s} d s+\frac{C}{\Gamma(q)} \int_{1}^{t_{m}}\left(\log \frac{t}{s}\right)^{q-1} \frac{\omega_{2}\left(s, R_{0}, R_{0}\right)}{s} d s \\
& +\Psi_{1}\left(\|x\|_{\left[t_{m}, T\right]},\|y\|_{\left[t_{m}, T\right]}\right) \frac{1}{\Gamma(p)} \int_{t_{m}}^{T}\left(\log \frac{T}{s}\right)^{p-1} \frac{k(s)}{s} d s,
\end{aligned}
$$

and consequently,

$$
\begin{aligned}
& \|x\|_{\left[t_{m}, T\right]} \\
& \leq \frac{B}{\Gamma(p)} \int_{1}^{t_{m}}\left(\log \frac{t}{s}\right)^{p-1} \frac{\omega_{1}\left(s, R_{0}, R_{0}\right)}{s} d s+\frac{C}{\Gamma(q)} \int_{1}^{t_{m}}\left(\log \frac{t}{s}\right)^{q-1} \frac{\omega_{2}\left(s, R_{0}, R_{0}\right)}{s} d s \\
& \quad+\Psi_{1}\left(\|x\|_{\left[t_{m}, T\right]},\|y\|_{\left[t_{m}, T\right]}\right) \frac{1}{\Gamma(p)} \int_{t_{m}}^{T}\left(\log \frac{T}{s}\right)^{p-1} \frac{k(s)}{s} d s .
\end{aligned}
$$

Similarly,

$$
\begin{aligned}
& |y(t)| \\
& \leq \leq \frac{B}{\Gamma(q)} \int_{1}^{t_{m}}\left(\log \frac{t}{s}\right)^{q-1} \frac{\omega_{2}\left(s, R_{0}, R_{0}\right)}{s} d s+\frac{D}{\Gamma(p)} \int_{1}^{t_{m}}\left(\log \frac{t}{s}\right)^{p-1} \frac{\omega_{1}\left(s, R_{0}, R_{0}\right)}{s} d s \\
& \quad+\Psi_{2}\left(\|x\|_{\left[t_{m}, T\right]},\|y\|_{\left[t_{m}, T\right]}\right) \frac{1}{\Gamma(q)} \int_{t_{m}}^{T}\left(\log \frac{t}{s}\right)^{q-1} \frac{k(s)}{s} d s
\end{aligned}
$$

and

$$
\begin{aligned}
& \|y\|_{\left[t_{m}, T\right]} \\
& \leq \frac{B}{\Gamma(q)} \int_{1}^{t_{m}}\left(\log \frac{t}{s}\right)^{q-1} \frac{\omega_{2}\left(s, R_{0}, R_{0}\right)}{s} d s+\frac{D}{\Gamma(p)} \int_{1}^{t_{m}}\left(\log \frac{t}{s}\right)^{p-1} \frac{\omega_{1}\left(s, R_{0}, R_{0}\right)}{s} d s \\
& \quad+\Psi_{2}\left(\|x\|_{\left[t_{m}, T\right]},\|y\|_{\left[t_{m}, T\right]}\right) \frac{1}{\Gamma(q)} \int_{t_{m}}^{T}\left(\log \frac{t}{s}\right)^{q-1} \frac{k(s)}{s} d s .
\end{aligned}
$$

Then we have

$$
\begin{aligned}
\|x\|_{\left[t_{m}, T\right]}+\|y\|_{\left[t_{m}, T\right]} & \\
\leq & \frac{B+D}{\Gamma(p)} \int_{1}^{t_{m}}\left(\log \frac{t}{s}\right)^{p-1} \frac{\omega_{1}\left(s, R_{0}, R_{0}\right)}{s} d s+\frac{B+C}{\Gamma(q)} \int_{1}^{t_{m}}\left(\log \frac{t}{s}\right)^{q-1} \frac{\omega_{2}\left(s, R_{0}, R_{0}\right)}{s} d s \\
& +\Psi_{1}\left(\|x\|_{\left[t_{m}, T\right]},\|y\|_{\left[t_{m}, T\right]}\right) \frac{1}{\Gamma(p)} \int_{t_{m}}^{T}\left(\log \frac{T}{s}\right)^{p-1} \frac{k(s)}{s} d s \\
& +\Psi_{2}\left(\|x\|_{\left[t_{m}, T\right]},\|y\|_{\left[t_{m}, T\right]}\right) \frac{1}{\Gamma(q)} \int_{t_{m}}^{T}\left(\log \frac{t}{s}\right)^{q-1} \frac{k(s)}{s} d s \\
= & \Lambda_{1}+\Lambda_{2}
\end{aligned}
$$


and

$$
\frac{\|x\|_{\left[t_{m}, T\right]}+\|y\|_{\left[t_{m}, T\right]}}{\Lambda_{1}+\Lambda_{2}} \leq 1
$$

Now $\left(\mathrm{H}_{4}\right)$ implies that there exists $R^{*}>0$ such that for all $R>R^{*}$, we have

$$
\frac{R}{\Lambda_{1}+\Lambda_{2}}>1
$$

Comparing inequalities (22) and (23), we find that

$$
\|x\|_{\left[t_{m}, T\right]}+\|y\|_{\left[t_{m}, T\right]} \leq R^{*} .
$$

Let $\gamma=\max \left\{R_{0}, R^{*}\right\}$. We have $\|x\|_{[1, T]} \leq \gamma$ and $\|y\|_{[1, T]} \leq \gamma$. It follows from $\left(H_{1}\right)$ that there exist $\sigma_{\gamma}, \varrho_{\gamma} \in L^{1}\left([1, T], \mathbb{R}^{+}\right)$such that $|f(t, x, y)| \leq \sigma_{\gamma}$ and $|g(t, x, y)| \leq \varrho_{\gamma}$ for almost all $t \in[1, T]$.

The operator $\mathcal{F}: \bar{B}_{\gamma} \rightarrow C[1, T]$ is continuous and completely continuous. Indeed, $\mathcal{F}$ is continuous in view of $\left(H_{1}\right)$, and for complete continuity, we remark that the operator is uniformly bounded as

$$
\begin{aligned}
\left|\mathcal{F}_{1}(x(t), y(t))\right|= & \frac{1}{\Gamma(p)} \int_{1}^{t}\left(\log \frac{t}{s}\right)^{p-1} \frac{f(s, x(s), y(s))}{s} d s \\
& -\eta\left[\sum_{j=1}^{m} \alpha_{j} \frac{1}{\Gamma(q)} \int_{1}^{t_{j}}\left(\log \frac{t_{j}}{s}\right)^{q-1} \frac{g(s, x(s), y(s))}{s} d s\right. \\
& \left.-\sum_{j=1}^{m} \alpha_{j} \sum_{j=1}^{m} \beta_{j} \frac{1}{\Gamma(p)} \int_{1}^{t_{j}}\left(\log \frac{t_{j}}{s}\right)^{p-1} \frac{f(s, x(s), y(s))}{s} d s\right] \mid \\
\leq & \frac{1}{\Gamma(p)} \int_{1}^{T}\left(\log \frac{T}{s}\right)^{p-1} \frac{\sigma_{\gamma}}{s} d s \\
& +|\eta|\left[\sum_{j=1}^{m}\left|\alpha_{j}\right| \frac{1}{\Gamma(q)} \int_{1}^{T}\left(\log \frac{T}{s}\right)^{q-1} \frac{\varrho_{\gamma}}{s} d s\right. \\
& \left.+\sum_{j=1}^{m}\left|\alpha_{j}\right| \sum_{j=1}^{m}\left|\beta_{j}\right| \frac{1}{\Gamma(p)} \int_{1}^{T}\left(\log \frac{T}{s}\right)^{p-1} \frac{\sigma_{\gamma}}{s} d s\right] \\
\leq & B \frac{1}{\Gamma(p)} \int_{1}^{T}\left(\log \frac{T}{s}\right)^{p-1} \frac{\sigma_{\gamma}}{s} d s+C \frac{1}{\Gamma(q)} \int_{1}^{T}\left(\log \frac{T}{s}\right)^{q-1} \frac{\varrho_{\gamma}}{s} d s .
\end{aligned}
$$

Similarly,

$$
\left|\mathcal{F}_{2}(x(t), y(t))\right| \leq \frac{B}{\Gamma(q)} \int_{1}^{T}\left(\log \frac{T}{s}\right)^{q-1} \frac{\varrho_{\gamma}}{s} d s+\frac{D}{\Gamma(p)} \int_{1}^{T}\left(\log \frac{T}{s}\right)^{p-1} \frac{\sigma_{\gamma}}{s} d s
$$

and it is equicontinuous since

$$
\begin{aligned}
& \left|\mathcal{F}_{1}\left(x\left(v_{2}\right), y\left(v_{2}\right)\right)-\mathcal{F}_{1}\left(x\left(v_{1}\right), y\left(v_{1}\right)\right)\right| \\
& \quad=\mid \frac{1}{\Gamma(p)} \int_{1}^{v_{1}}\left[\left(\log \frac{v_{2}}{s}\right)^{p-1}-\left(\log \frac{\nu_{1}}{s}\right)^{p-1}\right] \frac{f(s, x(s), y(s))}{s} d s
\end{aligned}
$$




$$
\begin{aligned}
& +\frac{1}{\Gamma(p)} \int_{\nu_{1}}^{\nu_{2}}\left(\log \frac{\nu_{2}}{s}\right)^{p-1} \frac{f(s, x(s), y(s))}{s} d s \mid \\
\leq & \mid \frac{1}{\Gamma(p)} \int_{1}^{\nu_{1}}\left[\left(\log \frac{\nu_{2}}{s}\right)^{p-1}-\left(\log \frac{\nu_{1}}{s}\right)^{p-1}\right] \frac{\sigma_{\gamma}}{s} d s \\
+ & \frac{1}{\Gamma(p)} \int_{\nu_{1}}^{\nu_{2}}\left(\log \frac{\nu_{2}}{s}\right)^{p-1} \frac{\sigma_{\gamma}}{s} d s \mid .
\end{aligned}
$$

Similarly,

$$
\begin{aligned}
\left|\mathcal{F}_{2}\left(x\left(v_{2}\right), y\left(v_{2}\right)\right)-\mathcal{F}_{2}\left(x\left(v_{1}\right), y\left(v_{1}\right)\right)\right| \\
\leq \mid \frac{1}{\Gamma(q)} \int_{1}^{v_{1}}\left[\left(\log \frac{v_{2}}{s}\right)^{q-1}-\left(\log \frac{v_{1}}{s}\right)^{q-1}\right] \frac{\varrho_{\gamma}}{s} d s \\
\quad+\frac{1}{\Gamma(q)} \int_{v_{1}}^{v_{2}}\left(\log \frac{\nu_{2}}{s}\right)^{q-1} \frac{\varrho_{\gamma}}{s} d s \mid,
\end{aligned}
$$

where $1<v_{1}<v_{2}<T$. Hence, by the Leray-Schauder alternative [24], we deduce that the operator $\mathcal{F}$ has a fixed point in $B_{\gamma}$, which is a solution of problem (2)-(3). The proof is completed.

\section{Further existence and uniqueness results}

In the next theorem, we prove the uniqueness of solutions for problem (2)-(3) via Banach's fixed point theorem.

Theorem 2 Let $f, g:[1, T] \times \mathbb{R}^{2} \rightarrow \mathbb{R}$ be jointly continuous functions and satisfy the assumption

$\left(K_{1}\right)$ There exist constants $L_{1}, L_{2}>0$ such that $\forall t \in[1, T]$ and $x_{\epsilon}, y_{\epsilon} \in \mathbb{R}, \epsilon=1,2$,

$$
\begin{aligned}
& \left|f\left(t, x_{1}, y_{1}\right)-f\left(t, x_{2}, y_{2}\right)\right| \leq L_{1}\left(\left|x_{1}-y_{1}\right|+\left|x_{2}-y_{2}\right|\right), \\
& \left|g\left(t, x_{1}, y_{1}\right)-g\left(t, x_{2}, y_{2}\right)\right| \leq L_{2}\left(\left|x_{1}-y_{1}\right|+\left|x_{2}-y_{2}\right|\right) .
\end{aligned}
$$

Then, problem (2)-(3) has a unique solution on $[1, T]$ if $\Lambda<1$, where

$$
\Lambda=L_{1}\left(m_{1}+n_{1}\right)+L_{2}\left(m_{2}+n_{2}\right)
$$

Proof Let us define $M_{1}, M_{2}$ as finite numbers given by

$$
M_{1}=\sup _{t \in[1, T]}|f(t, 0,0)|, \quad M_{2}=\sup _{t \in[1, T]}|g(t, 0,0)|,
$$

and show that $\mathcal{F} B_{r} \subset B_{r}$, where $B_{r}=\left\{(x, y) \in C[1, T]^{2}:\|(x, y)\| \leq r\right\}$ with

$$
r>\frac{M_{1}\left(m_{1}+n_{2}\right)+M_{2}\left(m_{2}+n_{2}\right)}{1-\Lambda} .
$$

For any $(x, y) \in B_{r}, t \in[1, T]$, using $\left(K_{1}\right)$, we get

$$
|f(t, x, y)|=|f(t, x, y)-f(t, 0,0)+f(t, 0,0)|
$$




$$
\begin{aligned}
& \leq|f(t, x, y)-f(t, 0,0)|+|f(t, 0,0)| \\
& \leq L_{1}(|x(t)|+|y(t)|)+M_{1} \leq L_{1}(\|x\|+\|y\|)+M_{1} \leq L_{1} r+M_{1} .
\end{aligned}
$$

Similarly, we can establish that $|g(t, x, y)| \leq L_{2} r+M_{2}$. Then

$$
\begin{aligned}
\left\|\mathcal{F}_{1}(x, y)\right\| & \\
\leq & \frac{1}{\Gamma(p)} \int_{1}^{t}\left(\log \frac{t}{s}\right)^{p-1} \frac{|f(s, x(s), y(s))|}{s} d s \\
& +|\eta|\left[\sum_{j=1}^{m}\left|\alpha_{j}\right| \frac{1}{\Gamma(q)} \int_{1}^{t_{j}}\left(\log \frac{t_{j}}{s}\right)^{q-1} \frac{|g(s, x(s), y(s))|}{s} d s\right. \\
& \left.+\sum_{j=1}^{m}\left|\alpha_{j}\right| \sum_{j=1}^{m}\left|\beta_{j}\right| \frac{1}{\Gamma(p)} \int_{1}^{t_{j}}\left(\log \frac{t_{j}}{s}\right)^{p-1} \frac{|f(s, x(s), y(s))|}{s} d s\right] \\
\leq & \frac{\left(L_{1} r+M_{1}\right)}{\Gamma(p)}\left[\int_{1}^{t}\left(\log \frac{t}{s}\right)^{p-1} \frac{d s}{s}+|\eta| \sum_{j=1}^{m}\left|\alpha_{j}\right| \sum_{j=1}^{m}\left|\beta_{j}\right| \int_{1}^{t_{j}}\left(\log \frac{t_{j}}{s}\right)^{p-1} \frac{d s}{s}\right] \\
& +\frac{\left(L_{2} r+M_{2}\right)}{\Gamma(q)}\left[|\eta| \sum_{j=1}^{m}\left|\alpha_{j}\right| \int_{1}^{t_{j}}\left(\log \frac{t_{j}}{s}\right)^{q-1} \frac{d s}{s}\right] \\
\leq & \frac{\left(L_{1} r+M_{1}\right)}{\Gamma(p+1)}\left[(\log T)^{p}+|\eta|(\log T)^{p} \sum_{j=1}^{m}\left|\alpha_{j}\right| \sum_{j=1}^{m}\left|\beta_{j}\right|\right] \\
& +\frac{\left(L_{2} r+M_{2}\right)}{\Gamma(q+1)}\left[|\eta|(\log T)^{q} \sum_{j=1}^{m}\left|\alpha_{j}\right|\right] \\
= & \left(L_{1} m_{1}+L_{2} m_{2}\right) r+m_{1} M_{1}+m_{2} M_{2} .
\end{aligned}
$$

Similarly, we can find that

$$
\left\|\mathcal{F}_{2}(x, y)\right\| \leq\left(L_{1} n_{1}+L_{2} n_{2}\right) r+n_{1} M_{1}+n_{2} M_{2} .
$$

Consequently, in view of (24), we get

$$
\|\mathcal{F}(x, y)\| \leq \Lambda r+M_{1}\left(m_{1}+n_{1}\right)+M_{2}\left(m_{2}+n_{2}\right) \leq r,
$$

which shows that $\mathcal{F} B_{r} \subset B_{r}$.

Now, for any $\left(x_{1}, y_{1}\right),\left(x_{2}, y_{2}\right) \in C[1, T]^{2}$ and for each $t \in[1, T]$, we obtain

$$
\begin{aligned}
& \left\|\mathcal{F}_{1}\left(x_{1}, y_{1}\right)-\mathcal{F}_{1}\left(x_{2}, y_{2}\right)\right\| \\
& \leq \frac{1}{\Gamma(p)} \int_{1}^{t}\left(\log \frac{t}{s}\right)^{p-1}\left|f\left(s, x_{1}(s), y_{1}(s)\right)-f\left(s, x_{2}(s), y_{2}(s)\right)\right| \frac{d s}{s} \\
& \quad+|\eta|\left[\sum_{j=1}^{m}\left|\alpha_{j}\right| \frac{1}{\Gamma(q)} \int_{1}^{t_{j}}\left(\log \frac{t_{j}}{s}\right)^{q-1}\left|g\left(s, x_{1}(s), y_{1}(s)\right)-g\left(s, x_{2}(s), y_{2}(s)\right)\right| \frac{d s}{s}\right. \\
& \left.\quad+\sum_{j=1}^{m}\left|\alpha_{j}\right| \sum_{j=1}^{m}\left|\beta_{j}\right| \frac{1}{\Gamma(p)} \int_{1}^{t_{j}}\left(\log \frac{t_{j}}{s}\right)^{p-1}\left|f\left(s, x_{1}(s), y_{1}(s)\right)-f\left(s, x_{2}(s), y_{2}(s)\right)\right| \frac{d s}{s}\right]
\end{aligned}
$$




$$
\begin{aligned}
\leq & L_{1}\left(\left\|x_{1}-x_{2}\right\|+\left\|y_{1}-y_{2}\right\|\right) \\
& \times \frac{1}{\Gamma(p)}\left[\int_{1}^{t}\left(\log \frac{t}{s}\right)^{p-1} \frac{d s}{s}+|\eta| \sum_{j=1}^{m}\left|\alpha_{j}\right| \sum_{j=1}^{m}\left|\beta_{j}\right| \int_{1}^{t_{j}}\left(\log \frac{t_{j}}{s}\right)^{p-1} \frac{d s}{s}\right] \\
& +L_{2}\left(\left\|x_{1}-x_{2}\right\|+\left\|y_{1}-y_{2}\right\|\right) \frac{1}{\Gamma(q)}\left[|\eta| \sum_{j=1}^{m}\left|\alpha_{j}\right| \int_{1}^{t_{j}}\left(\log \frac{t_{j}}{s}\right)^{q-1} \frac{d s}{s}\right] \\
\leq & L_{1}\left(\left\|x_{1}-x_{2}\right\|+\left\|y_{1}-y_{2}\right\|\right) \frac{1}{\Gamma(p+1)}\left[(\log T)^{p}+|\eta|(\log T)^{p} \sum_{j=1}^{m}\left|\alpha_{j}\right| \sum_{j=1}^{m}\left|\beta_{j}\right|\right] \\
& +L_{2}\left(\left\|x_{1}-x_{2}\right\|+\left\|y_{1}-y_{2}\right\|\right) \frac{1}{\Gamma(q+1)}\left[|\eta|(\log T)^{q} \sum_{j=1}^{m}\left|\alpha_{j}\right|\right] \\
\leq & {\left[L_{1} m_{1}+L_{2} m_{2}\right]\left(\left\|x_{1}-x_{2}\right\|+\left\|y_{1}-y_{2}\right\|\right) . }
\end{aligned}
$$

Similarly, we find that

$$
\left\|\mathcal{F}_{2}\left(x_{1}, y_{1}\right)-\mathcal{F}_{2}\left(x_{2}, y_{2}\right)\right\| \leq\left[L_{1} n_{1}+L_{2} n_{2}\right]\left(\left\|x_{1}-x_{2}\right\|+\left\|y_{1}-y_{2}\right\|\right) .
$$

Consequently, we get

$$
\left\|\mathcal{F}\left(x_{1}, y_{1}\right)-\mathcal{F}\left(x_{2}, y_{2}\right)\right\| \leq \Lambda\left(\left\|x_{1}-x_{2}\right\|+\left\|y_{1}-y_{2}\right\|\right),
$$

which, by condition (24), implies that the operator $\mathcal{F}$ is a contraction. Hence the conclusion of Banach fixed point theorem applies, and consequently there exists a unique solution for the problem (2)-(3). The proof is complete.

In the following result, we make use of the Leray-Schauder nonlinear alternative.

\section{Theorem 3 Assume that}

$\left(O_{1}\right) f, g:[1, T] \times \mathbb{R}^{2} \rightarrow \mathbb{R}$ are jointly continuous functions;

$\left(O_{2}\right)$ There exist functions $k_{1}, k_{2} \in L^{1}\left([1, T], \mathbb{R}^{+}\right)$and nondecreasing functions $\Omega_{1}, \Omega_{2}$, $\Phi_{1}, \Phi_{2}: \mathbb{R}^{+} \rightarrow \mathbb{R}^{+}$such that $|f(t, x, y)| \leq k_{1}(t)\left[\Omega_{1}(\|x\|)+\Phi_{1}(\|y\|)\right],|g(t, x, y)| \leq$ $k_{2}(t)\left[\Omega_{2}(\|x\|)+\Phi_{2}(\|y\|)\right]$, for all $(t, x, y) \in[1, T] \times \mathbb{R}^{2}$.

$\left(\mathrm{O}_{3}\right)$ There exists a constant $S>0$ such that

$$
\frac{S}{\left(l_{1}+l_{4}\right)\left[\Omega_{1}(S)+\Phi_{1}(S)\right]+\left(l_{2}+l_{3}\right)\left[\Omega_{2}(S)+\Phi_{2}(S)\right]}>1,
$$

where $h_{i}, i=1,2,3,4$ are defined by (11).

Then problem (2)-(3) has at least one solution on $[1, T]$.

Proof We show the boundedness of the set of all solutions to equations $(x, y)=\lambda \mathcal{F}(x, y)$ for $\lambda \in[0,1]$. For that, let $(x, y)$ be a solution of $(x, y)=\lambda \mathcal{F}(x, y)$ for $\lambda \in[0,1]$. Then, for $t \in[1, T]$, we have

$$
\begin{aligned}
|x(t)| & =\left|\lambda \mathcal{F}_{1}(x, y)(t)\right| \\
& =\lambda \mid \frac{1}{\Gamma(p)} \int_{1}^{t}\left(\log \frac{t}{s}\right)^{p-1} \frac{f(s, x(s), y(s))}{s} d s
\end{aligned}
$$




$$
\begin{aligned}
& -\eta\left[\sum_{j=1}^{m} \alpha_{j} \frac{1}{\Gamma(q)} \int_{1}^{t_{j}}\left(\log \frac{t_{j}}{s}\right)^{q-1} \frac{g(s, x(s), y(s))}{s} d s\right. \\
& \left.-\sum_{j=1}^{m} \alpha_{j} \sum_{j=1}^{m} \beta_{j} \frac{1}{\Gamma(p)} \int_{1}^{t_{j}}\left(\log \frac{t_{j}}{s}\right)^{p-1} \frac{f(s, x(s), y(s))}{s} d s\right] \mid \\
\leq & \frac{1}{\Gamma(p)} \int_{1}^{T}\left(\log \frac{t}{s}\right)^{p-1} \frac{k_{1}(s)\left[\Omega_{1}(\|x\|)+\Phi_{1}(\|y\|)\right]}{s} d s \\
& +|\eta|\left[\sum_{j=1}^{m}\left|\alpha_{j}\right| \frac{1}{\Gamma(q)} \int_{1}^{t_{j}}\left(\log \frac{t_{j}}{s}\right)^{q-1} \frac{k_{2}(s)\left[\Omega_{2}(\|x\|)+\Phi_{2}(\|y\|)\right]}{s} d s\right. \\
& \left.+\sum_{j=1}^{m}\left|\alpha_{j}\right| \sum_{j=1}^{m}\left|\beta_{j}\right| \frac{1}{\Gamma(p)} \int_{1}^{t_{j}}\left(\log \frac{t_{j}}{s}\right)^{p-1} \frac{k_{1}(s)\left[\Omega_{1}(\|x\|)+\Phi_{1}(\|y\|)\right]}{s} d s\right] \\
\leq & B\left[\Omega_{1}(\|x\|)+\Phi_{1}(\|y\|)\right] \frac{1}{\Gamma(p)} \int_{1}^{T}\left(\log \frac{T}{s}\right)^{p-1} \frac{k_{1}(s)}{s} d s \\
& +C\left[\Omega_{2}(\|x\|)+\Phi_{2}(\|y\|)\right] \frac{1}{\Gamma(q)} \int_{1}^{T}\left(\log \frac{T}{s}\right)^{q-1} \frac{k_{2}(s)}{s} d s \\
\leq & l_{1}\left[\Omega_{1}(\|x\|)+\Phi_{1}(\|y\|)\right]+l_{2}\left[\Omega_{2}(\|x\|)+\Phi_{2}(\|y\|)\right] .
\end{aligned}
$$

Similarly, we have

$$
|y(t)| \leq l_{3}\left[\Omega_{2}(\|x\|)+\Phi_{2}(\|y\|)\right]+l_{4}\left[\Omega_{1}(\|x\|)+\Phi_{1}(\|y\|)\right] .
$$

For each $t \in[1, T]$, we obtain

$$
\|x\| \leq l_{1}\left[\Omega_{1}(\|x\|)+\Phi_{1}(\|y\|)\right]+l_{2}\left[\Omega_{2}(\|x\|)+\Phi_{2}(\|y\|)\right]
$$

and

$$
\|y\| \leq l_{3}\left[\Omega_{2}(\|x\|)+\Phi_{2}(\|y\|)\right]+l_{4}\left[\Omega_{1}(\|x\|)+\Phi_{1}(\|y\|)\right] .
$$

Hence we obtain

$$
\begin{aligned}
\|(x, y)\| & =\|x\|+\|y\| \\
& \leq\left(l_{1}+l_{4}\right)\left[\Omega_{1}(\|x\|)+\Phi_{1}(\|y\|)\right]+\left(l_{2}+l_{3}\right)\left[\Omega_{2}(\|x\|)+\Phi_{2}(\|y\|)\right],
\end{aligned}
$$

which implies that

$$
\frac{\|(x, y)\|}{\left(l_{1}+l_{4}\right)\left[\Omega_{1}(\|x\|)+\Phi_{1}(\|y\|)\right]+\left(l_{2}+l_{3}\right)\left[\Omega_{2}(\|x\|)+\Phi_{2}(\|y\|)\right]} \leq 1 .
$$

In view of $\left(O_{3}\right)$, there exists $S$ such that $(x, y) \neq S$. Let us set

$$
U=\{(x, y) \in X \times Y:\|(x, y)\|<S\} .
$$

As in the proof (last step) of Theorem 1, it can be shown that the operator $\mathcal{F}: U \rightarrow X$ is continuous and completely continuous. From the choice of $U$, there is no $(x, y) \in \partial U$ 
such that $(x, y)=\lambda \mathcal{F}(x, y)$ for some $\lambda \in(0,1)$. Consequently, by the nonlinear alternative of Leray-Schauder, we deduce that $\mathcal{F}$ has a fixed point $(x, y) \in U$, which is a solution of the problem (2)-(3). This completes the proof.

Example 1 Consider the following Hadamard-type nonlocal problem:

$$
\left\{\begin{array}{l}
{ }^{H} D^{3 / 14} x(t)=f(t, x(t), y(t)), \quad 1<t<e, \\
{ }^{H} D^{3 / 17} y(t)=g(t, x(t), y(t)),
\end{array}\right.
$$

subject to the initial conditions:

$$
\left\{\begin{array}{l}
x(1)+\sum_{j=1}^{4} \alpha_{j} y\left(t_{j}\right)=0, \\
y(1)+\sum_{j=1}^{4} \beta_{j} x\left(t_{j}\right)=0 .
\end{array}\right.
$$

Here, $p=3 / 14, q=3 / 17, T=e, \alpha_{1}=1 / 3, \alpha_{2}=1 / 9, \alpha_{3}=1 / 27, \alpha_{4}=1 / 81, \beta_{1}=2 / 3, \beta_{2}=4 / 9$, $\beta_{3}=8 / 27, \beta_{4}=16 / 81, t_{1}=5 / 4, t_{2}=3 / 2, t_{3}=7 / 4$, and $t_{4}=2$. With the given data, the values of $B, C, D, m_{1}, m_{2}, n_{1}$, and $n_{2}$ defined by (11) are found to be $\eta=6561 / 4691, B=6561 / 4691$, $C=3240 / 4691, D=10530 / 4691, m_{1} \approx 1.529394, m_{2} \approx 0.746878, n_{1} \approx 1.512428$, and $n_{2} \approx$ 2.454584. In order to illustrate Theorem 2 , we take

$$
\begin{aligned}
& f(t, x(t), y(t))=\frac{1}{19 \sqrt{168+t^{4}}}\left(\frac{|x(t)|}{1+|x(t)|}+\tan ^{-1} y(t)\right)+\cos 2 t, \\
& g(t, x(t), y(t))=\frac{1}{7 \sqrt{323+t^{2}}}\left(x(t)+\tan ^{-1} x(t)\right)+\frac{1}{\sqrt{676}+99+t^{2}}\left(\frac{|y(t)|}{1+|y(t)|}+\sin 2 t\right) .
\end{aligned}
$$

Clearly, $L_{1}=1 / 247$ as $\left|f\left(t, x_{1}, y_{1}\right)-f\left(t, x_{2}, y_{2}\right)\right| \leq \frac{1}{247}\left(\left|x_{1}-y_{1}\right|+\left|y_{1}-y_{2}\right|\right)$ and $L_{2}=1 / 126$ as $\left|g\left(t, x_{1}, y_{1}\right)-g\left(t, x_{2}, y_{2}\right)\right| \leq \frac{1}{126}\left(\left|x_{1}-y_{1}\right|+\left|y_{1}-y_{2}\right|\right)$. Using the given data, we have $\Lambda \approx$ $0.037723<1$. Obviously, the hypotheses of Theorem 2 are satisfied. Hence, by the conclusion of Theorem 2, there is a unique solution for the problem $(25)-(26)$ on $[1, e]$.

\section{Conclusions}

We have investigated the existence of solutions for a coupled system of Hadamard fractional differential equations with nonlocal coupled initial-multipoint conditions. The existence result is based on the idea of splitting the growth conditions into two subintervals, respectively containing the points involved in the nonlocal condition, and the rest of the interval. We apply Leray-Schauder alternative to prove this result. On the other hand, the uniqueness of solutions for the given problem is established by means of the contraction mapping principle. Our results are new and contribute to the existing literature on nonlocal nonlinear Hadamard-type boundary value problems.

\section{Acknowledgements}

This project was funded by the Deanship of Scientific Research (DSR), King Abdulaziz University, Jeddah, Saudi Arabia under grant no. (KEP-MSc-45-130-38). The authors, therefore, acknowledge with thanks DSR technical and financial support. 
Abbreviations

Not applicable.

\section{Availability of data and materials}

Not applicable.

\section{Competing interests}

The authors declare that they have no competing interests.

Authors' contributions

Each of the authors, BA, SKN, AA, and AFA, contributed equally to each part of this work. All authors read and approved the final manuscript.

\section{Author details}

${ }^{1}$ Nonlinear Analysis and Applied Mathematics (NAAM)-Research Group, Department of Mathematics, Faculty of Science, King Abdulaziz University, P.O. Box 80203, Jeddah 21589, Saudi Arabia. ${ }^{2}$ Department of Mathematics, University of loannina, 45110 loannina, Greece.

\section{Publisher's Note}

Springer Nature remains neutral with regard to jurisdictional claims in published maps and institutional affiliations.

Received: 19 November 2020 Accepted: 21 December 2020 Published online: 07 January 2021

\section{References}

1. Hilb, E.: Zur Theorie der Entwicklungen willkurlicher Funktionen nach Eigenfunktionen. Math. Z. 58, 1-9 (1918)

2. Bitsadze, A.V., Samarskii, A.A.: Some elementary generalizations of linear elliptic boundary value problems. Sov. Math. Dokl. 10, 398-400 (1969)

3. Balachandran, K., Uchiyama, K.: Existence of solutions of nonlinear integrodifferential equations of Sobolev type with nonlocal conditions in Banach spaces. Proc. Indian Acad. Sci. Math. Sci. 110, 225-232 (2000)

4. Byszewski, L.: Theorems about the existence and uniqueness of solutions of a semilinear evolution nonlocal Cauchy problem. J. Math. Anal. Appl. 162, 494-505 (1991)

5. Kilbas, A.A., Srivastava, H.M., Trujillo, J.J.: Theory and Applications of Fractional Differential Equations. North-Holland Mathematics Studies, vol. 204. Elsevier, Amsterdam (2006)

6. Podlubny, I.: Fractional Differential Equations. Academic Press, San Diego (1999)

7. Sabatier, J., Agrawal, O.P., Machado, J.A.T. (eds.): Advances in Fractional Calculus: Theoretical Developments and Applications in Physics and Engineering Springer, Dordrecht (2007)

8. Samko, S.G., Kilbas, A.A., Marichev, O.I.: Fractional Integrals and Derivatives, Theory and Applications. Gordon \& Breach, Yverdon (1993)

9. Lakshmikantham, V., Leela, S., Vasundhara Devi, J.: Theory of Fractional Dynamic Systems. Cambridge Academic Publishers, Cambridge (2009)

10. Ahmad, B., Alsaedi, A., Ntouyas, S.K., Tariboon, J.: Hadamard-Type Fractional Differential Equations, Inclusions and Inequalities. Springer, Berlin (2017)

11. Wang, G., Pei, K., Agarwal, R.P., Zhang, L., Ahmad, B.: Nonlocal Hadamard fractional boundary value problem with Hadamard integral and discrete boundary conditions on a half-line. J. Comput. Appl. Math. 343, 230-239 (2018)

12. Garra, R., Orsingher, E., Polito, F.: A note on Hadamard fractional differential equations with varying coefficients and their applications in probability. Mathematics 6, 4 (2018)

13. Saxena, R.K., Garra, R., Orsingher, E.: Analytical solution of space-time fractional telegraph-type equations involving Hilfer and Hadamard derivatives. Integral Transforms Spec. Funct. 27, 30-42 (2016)

14. Aljoudi, S., Ahmad, B., Nieto, J.J., Alsaedi, A.: A coupled system of Hadamard type sequential fractional differential equations with coupled strip conditions. Chaos Solitons Fractals 91, 39-46 (2016)

15. Tariboon, J., Ntouyas, S.K., Asawasamrit, S., Promsakon, C.: Positive solutions for Hadamard differential systems with fractional integral conditions on an unbounded domain. Open Math. 15, 645-666 (2017)

16. Aljoudi, S., Ahmad, B., Nieto, J.J., Alsaedi, A.: On coupled Hadamard type sequential fractional differential equations with variable coefficients and nonlocal integral boundary conditions. Filomat 31, 6041-6049 (2017)

17. Zhai, C., Wang, W., Li, H.: A uniqueness method to a new Hadamard fractional differential system with four-point boundary conditions. J. Inequal. Appl. 2018, Paper No. 207, 16 pp. (2018)

18. Du, X., Meng, Y., Pang, H.: Iterative positive solutions to a coupled Hadamard-type fractional differential system on infinite domain with the multistrip and multipoint mixed boundary conditions. J. Funct. Spaces 2020, Article ID 6508075 (2020)

19. Ma, L.: On the kinetics of Hadamard-type fractional differential systems. Fract. Calc. Appl. Anal. 23, 553-570 (2020)

20. Ahmad, B., Ntouyas, S.K.: Nonlocal initial value problems for Hadamard-type fractional differential equations and inclusions. Rocky Mt. J. Math. 48, 1043-1068 (2018)

21. Boucherif, A., Precup, R.: On the nonlocal initial value problem for first order differential equations. Fixed Point Theory 4(2), 205-212 (2003)

22. Anderson, D., Boucherif, A.: Nonlocal initial value problem for first-order dynamic equations on time scales. Dyn. Contin. Discrete Impuls. Syst., Ser. A Math. Anal. 16, 222-226 (2009)

23. Boucherif, A., Ntouyas, S.K.: Nonlocal initial value problems for first order fractional differential equations. Dyn. Syst. Appl. 20, 247-260 (2011)

24. Granas, A., Dugundji, J.: Fixed Point Theory. Springer, New York (2003) 